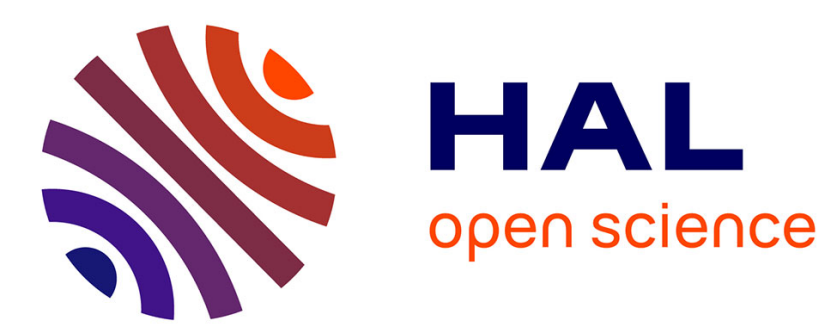

\title{
The Dual Jacobian of a Generalised Hyperbolic Tetrahedron, and Volumes of Prisms
}

Alexander Kolpakov, Jun Murakami

\section{To cite this version:}

Alexander Kolpakov, Jun Murakami. The Dual Jacobian of a Generalised Hyperbolic Tetrahedron, and Volumes of Prisms. Tokyo Journal of Mathematics, 2016, 39, pp.45 - 67. 10.3836/tjm/1471873312. hal-01396110

\section{HAL Id: hal-01396110 https://hal.science/hal-01396110}

Submitted on 14 Nov 2016

HAL is a multi-disciplinary open access archive for the deposit and dissemination of scientific research documents, whether they are published or not. The documents may come from teaching and research institutions in France or abroad, or from public or private research centers.
L'archive ouverte pluridisciplinaire HAL, est destinée au dépôt et à la diffusion de documents scientifiques de niveau recherche, publiés ou non, émanant des établissements d'enseignement et de recherche français ou étrangers, des laboratoires publics ou privés. 


\title{
The dual Jacobian of a generalised hyperbolic tetrahedron, and volumes of prisms
}

\author{
Alexander Kolpakov \\ Jun Murakami
}

\begin{abstract}
We derive an analytic formula for the dual Jacobian matrix of a generalised hyperbolic tetrahedron. Two cases are considered: a mildly truncated and a prism truncated tetrahedron. The Jacobian for the latter arises as an analytic continuation of the former, that falls in line with a similar behaviour of the corresponding volume formulae.

Also, we obtain a volume formula for a hyperbolic $n$-gonal prism: the proof requires the above mentioned Jacobian, employed in the analysis of the edge lengths behaviour of such a prism, needed later for the Schläfli formula.
\end{abstract}

Key words: hyperbolic polyhedron, Gram matrix, volume.

\section{Introduction}

Let $T$ be a generalised hyperbolic tetrahedron (in the sense of $[19,22]$ ) depicted in Fig. 1. If the truncating planes associated with its ultra-ideal vertices do not intersect, we call such a tetrahedron mildly truncated, otherwise we call it intensely truncated. If only two of them intersect, we call such a tetrahedron prism truncated [12]. Let us note that a prism truncated orthoscheme is, in fact, a Lambert cube [11].

The volumes of the tetrahedron and its truncations are of particular interest, since they are the simplest representatives of hyperbolic polyhedra. Over the last decade an extensive study produced a number of volume formulae suitable for analytic and numerical exploration [3, 5, 11, 12, 20, 22]. A similar study was done for the spherical tetrahedron $[14,17]$, which can be viewed as a natural counterpart of the hyperbolic one. Many analytic properties of the volume formula for a hyperbolic tetrahedron came into view concerning the Volume Conjecture [10, 18].

However, other geometric characteristics of a generalised hyperbolic tetrahedron $T$ are also important and bring some useful information. In particular, 


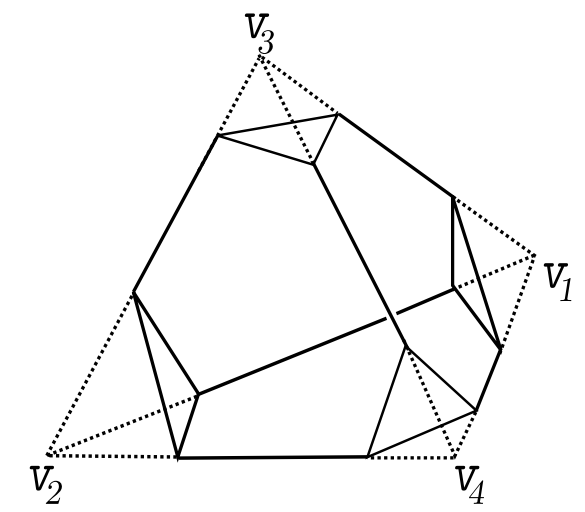

Figure 1: Generalised hyperbolic tetrahedron

$\operatorname{Jac}(T)$, the Jacobian of $T$, which is the Jacobian matrix of the edge length with respect to the dihedral angles, is such. This matrix enjoys many symmetries [15] and can be computed out of the Gram matrix of $T$ [7].

In the present paper, we consider $\operatorname{Jac}^{\star}(T)$, the dual Jacobian of a generalised hyperbolic tetrahedron $T$. By the dual Jacobian of $T$ we mean the Jacobian matrix of the dihedral angles with respect to the edge length. Such an object behaves nicely when $T$ undergoes both mild and intense truncation: the dual Jacobian of a prism truncated tetrahedron is an analytic continuation for that of a mildly doubly truncated one. Let us mention, that the respective volume formulae are also connected by an analytic continuation, in an analogous manner $[12,19]$.

As an application of our technique, we give a volume formula for a hyperbolic $n$-gonal prism, c.f. [4].

Acknowledgements. The authors gratefully acknowledge financial support provided by the Swiss National Science Foundation (SNSF project no. P300P2-151316) and the Japan Society for the Promotion of Science (Grant-in-Aid projects no. 25287014, no. 2561002 and Invitation Programs for Research project no. S-14021). The authors thank the anonymous referee for his/her careful reading of the manuscript and helpful comments.

\section{Preliminaries}

Let $T$ be a mildly truncated hyperbolic tetrahedron with vertices $\mathrm{v}_{k}, k \in$ $\{1,2,3,4\}$, edges $e_{i j}$ (connecting the vertices $\mathrm{v}_{i}$ and $\mathrm{v}_{j}$ ) with dihedral angles 
$a_{i j}$ and lengths $\ell_{i j}, i, j \in\{1,2,3,4\}, i<j$.

Depending on whether the vertex $\mathrm{v}_{k}$ is proper $\left(\mathrm{v}_{k} \in \mathbb{H}^{3}\right)$, ideal $\left(\mathrm{v}_{k} \in \partial \mathbb{H}^{3}\right)$ or ultra-ideal $\left(\mathrm{v}_{k}\right.$ defines a polar hyperplane as described in [21, Section 3], c.f. Theorem 3.2.12), let us set the quantity $\varepsilon_{k}$ to be $+1,0$ or -1 , respectively. For each vertex $\mathrm{v}_{i}$ of $T$ let us consider the face $F_{j k l}$ opposite to it, where $\{i, j, k, l\}=\{1,2,3,4\}$. The link $L\left(\mathrm{v}_{l}\right)$ of the vertex $\mathrm{v}_{l}$ is either a spherical triangle $\left(\varepsilon_{l}=+1\right)$, a Euclidean triangle $\left(\varepsilon_{l}=0\right)$ or a hyperbolic triangle $\left(\varepsilon_{l}=-1\right)$. Let us define the quantity $b_{j k}^{i}$ as follows:

$$
b_{j k}^{i}:=\left\{\begin{array}{l}
\text { the plane angle of } F_{j k l} \text { opposite to the edge } e_{j k}, \text { if } \varepsilon_{l}=+1 \\
\text { zero, if } \varepsilon_{l}=0 ; \\
\text { the length of the common perpendicular to the edges } e_{j l} \\
\text { and } e_{k l} \text { of } F_{j k l}, \text { if } \varepsilon_{l}=-1
\end{array}\right.
$$

Here, we consider the face $F_{j k l}$ as a generalised hyperbolic triangle, for which the trigonometric laws hold as described in $[2,9]$.

Let us also define a quantity $\mu_{j k}^{i}$ by means of the formula

$$
\mu_{j k}^{i}:=\int_{0}^{b_{j k}^{i}} \cos \left(\sqrt{\varepsilon_{l}} s\right) \mathrm{d} s .
$$

Let $\mu_{j k}^{\prime i}$ denote the derivative of $\mu_{j k}^{i}$ with respect to $b_{j k}^{i}$, which means that

$$
\mu_{j k}^{\prime i}=\cos \left(\sqrt{\varepsilon_{l}} b_{j k}^{i}\right)
$$

Let $\sigma_{k l}$ denote the following quantity associated with an edge $e_{k l}, k, l \in$ $\{1,2,3,4\}, k<l$,

$$
\sigma_{k l}:=\frac{1}{2} e^{\ell_{k l}}-\frac{1}{2} \varepsilon_{k} \varepsilon_{l} e^{-\ell_{k l}}
$$

Let $\sigma_{k l}^{\prime}$ denote the derivative of $\sigma_{k l}$ with respect to $\ell_{k l}$, so we have that

$$
\sigma_{k l}^{\prime}=\frac{1}{2} e^{\ell_{k l}}+\frac{1}{2} \varepsilon_{k} \varepsilon_{l} e^{-\ell_{k l}} .
$$

Let us define the momentum $M_{i}$ of the vertex $\mathrm{v}_{i}$ opposite to the face $F_{j k l}$, $\{i, j, k, l\}=\{1,2,3,4\}$ by the following equality (c.f. [6, VII.6]):

$$
M^{i}:=\mu_{j k}^{i} \mu_{j l}^{i} \sigma_{k l} \text {. }
$$

The quantity above is well defined grace to the following theorem. 
Theorem 1 (The Sine Law for faces) Let $F_{j k l}$ be the face of $T$ opposite to the vertex $\mathrm{v}_{i},\{i, j, k, l\}=\{1,2,3,4\}$. Then $F_{j k l}$ is a generalised hyperbolic triangle and the following equalities hold:

$$
\frac{\mu_{j k}^{i}}{\sigma_{j k}}=\frac{\mu_{j l}^{i}}{\sigma_{j l}}=\frac{\mu_{k l}^{i}}{\sigma_{k l}} .
$$

Let us also define the momentum $M_{j k l}$ of the face $F_{j k l}$ opposite to the vertex $\mathrm{v}_{i},\{i, j, k, l\}=\{1,2,3,4\}$ by setting (c.f. [6, VII.6])

$$
M_{j k l}:=\mu_{k l}^{j} \sin a_{i k} \sin a_{i l} .
$$

The quantity above is well defined, according to the following theorem.

Theorem 2 (The Sine Law for links) Let $\mathrm{v}_{i}$ be the vertex of $T$ opposite to the face $F_{j k l},\{i, j, k, l\}=\{1,2,3,4\}$. Then $L\left(\mathrm{v}_{i}\right)$ is either a spherical, a Euclidean or a hyperbolic triangle and the following equalities hold:

$$
\frac{\sin a_{i j}}{\mu_{k l}^{j}}=\frac{\sin a_{i k}}{\mu_{j l}^{k}}=\frac{\sin a_{i l}}{\mu_{j k}^{l}} .
$$

Both Theorem 1 and Theorem 2 are paraphrases of the spherical, Euclidean or hyperbolic sine laws (for a generalised hyperbolic triangle, see [9]). The following theorems are the cosine laws for a generalised hyperbolic triangle adopted to the notation of the present paper.

Theorem 3 (The first Cosine Law for faces) Let $F_{j k l}$ be the face of $T$ opposite to the vertex $\mathrm{v}_{i},\{i, j, k, l\}=\{1,2,3,4\}$. Then $F_{j k l}$ is a generalised hyperbolic triangle and the following equality holds:

$$
\sigma_{k l}^{\prime}=\frac{\mu_{k l}^{\prime i}+\mu_{j k}^{\prime i} \mu_{j l}^{\prime i}}{\mu_{j k}^{i} \mu_{j l}^{i}} .
$$

Theorem 4 (The second Cosine Law for faces) Let $F_{j k l}$ be the face of $T$ opposite to the vertex $\mathrm{v}_{i},\{i, j, k, l\}=\{1,2,3,4\}$. Then $F_{j k l}$ is a generalised hyperbolic triangle and the following equality holds:

$$
\mu_{j k}^{\prime i}=\frac{-\varepsilon_{l} \sigma_{j k}^{\prime}+\sigma_{j l}^{\prime} \sigma_{k l}^{\prime}}{\sigma_{j l} \sigma_{k l}} .
$$

Theorem 5 (The Cosine Law for links) Let $\mathrm{v}_{i}$ be the vertex of $T$ opposite to the face $F_{j k l},\{i, j, k, l\}=\{1,2,3,4\}$. Then $L\left(\mathrm{v}_{i}\right)$ is either a spherical, a Euclidean or a generalised hyperbolic triangle and the following equality holds:

$$
\mu_{k l}^{\prime j}=\frac{\cos a_{i j}+\cos a_{i k} \cos a_{i l}}{\sin a_{i k} \sin a_{i l}} .
$$




\section{Auxiliary lemmata}

In the present section we shall consider various partial derivatives of certain geometric quantities associated with either the faces or the vertex links of a generalised hyperbolic tetrahedron $T$. These derivatives will be used later on in the computation of the entries of $\operatorname{Jac}^{\star}(T)$.

Lemma 1 For $\{i, j, k, l\}=\{1,2,3,4\}$ we have

$$
\begin{gathered}
\frac{\partial \ell_{k l}}{\partial b_{k l}^{i}}=-\varepsilon_{j} \frac{\mu_{k l}^{i}}{M^{i}}, \\
\frac{\partial \ell_{k l}}{\partial b_{j k}^{i}}=-\sigma_{j l}^{\prime} \frac{\mu_{k l}^{i}}{M^{i}}, \quad \frac{\partial \ell_{k l}}{\partial b_{j l}^{i}}=-\sigma_{j k}^{\prime} \frac{\mu_{k l}^{i}}{M^{i}} .
\end{gathered}
$$

Proof. According to the definition of $\sigma_{k l}$, we have $\sigma_{k l}=0$ only in the following two cases: $\varepsilon_{k}=\varepsilon_{l}=+1$ and $\ell_{k l}=0$, or $\varepsilon_{k}=\varepsilon_{l}=-1$ and $\ell_{k l}=0$. In the former case, we have a degenerate tetrahedron with two proper vertices collapsing to one point. In the latter case the tetrahedron has two ultra-ideal vertices, whose polar planes are tangent at a point on the ideal boundary $\partial \mathbb{H}^{3}$. This is a limiting case, since in a generalised (mildly truncated) tetrahedron two polar planes never intersect or become tangent. Thus, we suppose that $\sigma_{k l} \neq 0$.

By taking derivatives on both sides of the first Cosine Law for faces, we get the following formulae:

$$
\sigma_{k l} \frac{\partial \ell_{k l}}{\partial b_{k l}^{i}}=\frac{\partial \sigma_{k l}^{\prime}}{\partial b_{k l}^{i}}=\frac{1}{\mu_{j k}^{i} \mu_{j l}^{i}} \frac{\partial \mu_{k l}^{\prime i}}{\partial b_{k l}^{i}}=-\varepsilon_{j} \frac{\mu_{k l}^{i}}{\mu_{j k}^{i} \mu_{j l}^{i}},
$$

since

$$
\frac{\partial \sigma_{k l}^{\prime}}{\partial b_{k l}^{i}}=\sigma_{k l} \frac{\partial \ell_{k l}}{\partial b_{k l}^{i}} \text { and } \frac{\partial \mu_{k l}^{\prime i}}{\partial b_{k l}^{i}}=-\varepsilon_{j} \mu_{k l}^{i}
$$

by a direct computation. This implies the first identity of the lemma.

Now we compute

$$
\begin{aligned}
\sigma_{k l} \frac{\partial \ell_{k l}}{\partial b_{j k}^{i}}= & \frac{\partial \sigma_{k l}^{\prime}}{\partial b_{j k}^{i}}=-\frac{\left(\left(\mu^{\prime i}{ }_{j k}\right)^{2}+\varepsilon_{l}\left(\mu_{j k}^{i}\right)^{2}\right) \mu_{j l}^{i} \mu_{j l}^{\prime i}+\mu_{j l}^{i} \mu_{j k}^{i} \mu_{k l}^{\prime i}}{\left(\mu_{j k}^{i} \mu_{j l}^{i}\right)^{2}}= \\
& -\frac{\mu_{j l}^{\prime i}+\mu^{\prime i}{ }_{j k} \mu_{k l}^{\prime i}}{\mu_{j k}^{i} \mu_{k l}^{i}} \cdot \frac{\mu_{k l}^{i}}{\mu_{j k}^{i} \mu_{j l}^{i}}=-\sigma_{j l}^{\prime} \frac{\mu_{k l}^{i}}{\mu_{j k}^{i} \mu_{j l}^{i}} .
\end{aligned}
$$


where we use the identity $\left(\mu_{j k}^{\prime i}\right)^{2}+\varepsilon_{l}\left(\mu_{j k}^{i}\right)^{2}=1$ and, as before, the fact that $\frac{\partial \mu_{j k}^{\prime i}}{\partial b_{j k}^{i}}=-\varepsilon_{l} \mu_{j k}^{i}$. Then the second identity follows. The third one is analogous to the second one under the permutation of the indices $k$ and $l$.

Lemma 2 For $\{i, j, k, l\}=\{1,2,3,4\}$ we have

$$
\begin{gathered}
\frac{\partial b_{k l}^{j}}{\partial a_{i j}}=\varepsilon_{i} \frac{\sin a_{i j}}{M_{j k l}} \\
\frac{\partial b_{k l}^{j}}{\partial a_{i k}}=\varepsilon_{i} \frac{\sin a_{i j}}{M_{j k l}} \mu_{j k}^{\prime l}, \quad \frac{\partial b_{k l}^{j}}{\partial a_{i l}}=\varepsilon_{i} \frac{\sin a_{i j}}{M_{j k l}} \mu_{j l}^{\prime k} .
\end{gathered}
$$

Proof. By taking derivatives on both sides of the Cosine Law for links, we get the following formulae:

$$
-\varepsilon_{i} \mu_{k l}^{j} \frac{\partial b_{k l}^{j}}{\partial a_{i j}}=\frac{\partial \mu_{k l}^{\prime j}}{\partial a_{i j}}=-\frac{\sin a_{i j}}{\sin a_{i k} \sin a_{i l}} .
$$

The first identity of the lemma follows.

Then we subsequently compute

$$
\begin{gathered}
-\varepsilon_{i} \mu_{k l}^{j} \frac{\partial b_{k l}^{j}}{\partial a_{i k}}=\frac{\partial \mu_{k l}^{\prime j}}{\partial a_{i k}}= \\
\mu_{j k}^{\prime l} \frac{\cos a_{i l}+\cos a_{i j} \cos a_{i k}}{\sin a_{i j} \sin a_{i k}} \frac{\sin a_{i j}}{\sin a_{i k} \sin a_{i l} \sin a_{i l}}=
\end{gathered}
$$

The second identity follows. The third one is analogous under the permutation of the indices $k$ and $l$.

Now we shall prove several identities that relate the principal minors $G_{i i}$, $i \in\{1,2,3,4\}$ of the Gram matrix $G:=G(T)$ of the tetrahedron $T$ with its face or vertex momenta.

Lemma 3 For $\{i, j, k, l\}=\{1,2,3,4\}$, we have that

$$
\operatorname{det} G_{i i}=\varepsilon_{i} M_{j k l}^{2} \text {. }
$$

Proof. Let us perform the computation for $G_{11}$ and other cases will follow by analogy. We have that

$$
\operatorname{det}\left(\begin{array}{ccc}
1 & -\cos a_{14} & -\cos a_{13} \\
-\cos a_{14} & 1 & -\cos a_{12} \\
-\cos a_{13} & -\cos a_{12} & 1
\end{array}\right)=
$$




$$
\begin{gathered}
=\operatorname{det}\left(\begin{array}{ccc}
1 & -\cos a_{14} & -\cos a_{13} \\
0 & \sin ^{2} a_{14} & -\mu_{34}^{\prime 2} \sin a_{13} \sin a_{14} \\
0 & -\mu_{34}^{\prime 2} \sin a_{13} \sin a_{14} & \sin ^{2} a_{13}
\end{array}\right)= \\
=\left(1-\left(\mu_{34}^{\prime 2}\right)^{2}\right) \sin ^{2} a_{13} \sin ^{2} a_{14}=\varepsilon_{1}\left(\mu_{34}^{2}\right)^{2} \sin ^{2} a_{13} \sin ^{2} a_{14}=\varepsilon_{1} M_{234}^{2} .
\end{gathered}
$$

By permuting the set $\{i, j, k, l\}=\{1,2,3,4\}$, one gets all other identities of the lemma.

Lemma 4 For $\{i, j, k, l\}=\{1,2,3,4\}$, we have that

$$
-\operatorname{det} G=\sin ^{2} a_{j k} \sin ^{2} a_{j l} \sin ^{2} a_{k l}\left(M^{i}\right)^{2} .
$$

Proof. Let us subsequently compute

$$
\begin{aligned}
& \operatorname{det} G=\operatorname{det}\left(\begin{array}{cccc}
1 & -\cos a_{34} & -\cos a_{24} & -\cos a_{23} \\
-\cos a_{34} & 1 & -\cos a_{14} & -\cos a_{13} \\
-\cos a_{24} & -\cos a_{14} & 1 & -\cos a_{12} \\
-\cos a_{23} & -\cos a_{13} & -\cos a_{12} & 1
\end{array}\right)= \\
& \operatorname{det}\left(\begin{array}{cccc}
1 & -\cos a_{34} & -\cos a_{24} & -\cos a_{23} \\
0 & \sin ^{2} a_{34} & -\mu^{\prime 1} \sin a_{24} \sin a_{34} & -\mu_{24}^{\prime 1} \sin a_{23} \sin a_{34} \\
0 & -\mu_{23}^{\prime 1} \sin a_{24} \sin a_{34} & \sin ^{2} a_{24} & -\mu^{\prime 1} \sin a_{23} \sin a_{24} \\
0 & -\mu^{\prime \prime}{ }_{24} \sin a_{23} \sin a_{34} & -\mu^{\prime \prime} \sin _{34} \sin a_{23} \sin a_{24} & \sin ^{2} a_{23}
\end{array}\right)= \\
& \sin ^{2} a_{23} \sin ^{2} a_{24} \sin ^{2} a_{34} \operatorname{det}\left(\begin{array}{ccc}
1 & -\mu_{23}^{\prime 1} & -\mu_{24}^{\prime 1} \\
-\mu_{23}^{\prime 1} & 1 & -\mu_{34}^{\prime 1} \\
-\mu_{24}^{\prime 1} & -\mu_{34}^{\prime 1} & 1
\end{array}\right)= \\
& \sin ^{2} a_{23} \sin ^{2} a_{24} \sin ^{2} a_{34} \operatorname{det}\left(\begin{array}{ccc}
1 & -\mu_{23}^{\prime 1} & -\mu_{24}^{\prime 1} \\
0 & \varepsilon_{4}\left(\mu_{23}^{1}\right)^{2} & -\sigma_{34}^{\prime} \mu_{23}^{1} \mu_{24}^{1} \\
0 & -\sigma_{34}^{\prime} \mu_{23}^{1} \mu_{24}^{1} & \varepsilon_{3}\left(\mu_{24}^{1}\right)^{2}
\end{array}\right)= \\
& \sin ^{2} a_{23} \sin ^{2} a_{24} \sin ^{2} a_{34}\left(\varepsilon_{3} \varepsilon_{4}-\left(\sigma_{34}^{\prime}\right)^{2}\right)\left(\mu_{23}^{1} \mu_{24}^{1}\right)^{2}= \\
& -\sin ^{2} a_{23} \sin ^{2} a_{24} \sin ^{2} a_{34}\left(M^{1}\right)^{2} .
\end{aligned}
$$

Here we used the Cosine Law for links in the second equality and the first Cosine Law for faces in the fourth equality. Also, we used the fact that for $\{i, j, k, l\}=\{1,2,3,4\}$ one has $1-\varepsilon_{l}\left(\mu_{j k}^{i}\right)^{2}=\left(\mu^{\prime i}{ }_{j k}\right)^{2}$ (in the third equality) and $\sigma_{i j}^{2}-\left(\sigma_{i j}^{\prime}\right)^{2}=\varepsilon_{i} \varepsilon_{j}$ (in the sixth equality). All other identities of the lemma follow by permuting the set $\{i, j, k, l\}=\{1,2,3,4\}$. 


\section{Dual Jacobian of a generalised hyperbolic tetra- hedron}

In this section we shall compute the entries of the dual Jacobian matrix $\operatorname{Jac}^{\star}(T)$ of a generalised hyperbolic tetrahedron $T$.

Theorem 6 Let $T$ be a generalised hyperbolic tetrahedron. Then

$$
\operatorname{Jac}^{\star}(T):=\frac{\partial\left(\ell_{12}, \ell_{13}, \ell_{14}, \ell_{23}, \ell_{24}, \ell_{34}\right)}{\partial\left(a_{12}, a_{13}, a_{14}, a_{23}, a_{24}, a_{34}\right)}=-\eta \mathscr{D} \mathscr{S} \mathscr{D},
$$

where

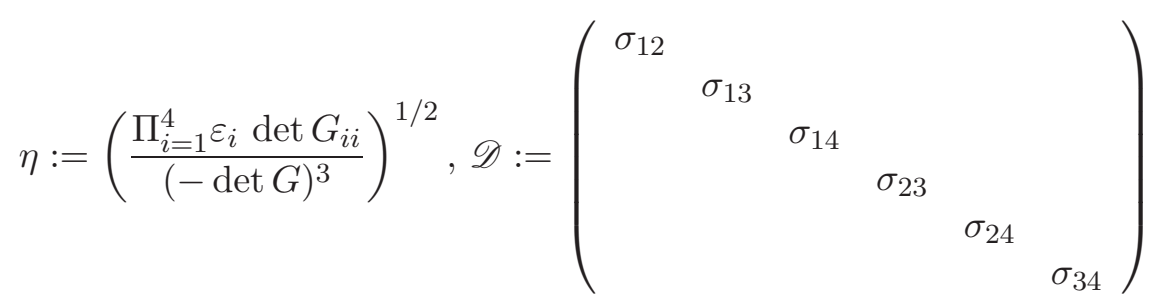

and

$$
\mathscr{S}:=\left(\begin{array}{cccccc}
\omega_{12} & \varepsilon_{1} \sigma_{14}^{\prime} & \varepsilon_{1} \sigma_{13}^{\prime} & \varepsilon_{2} \sigma_{24}^{\prime} & \varepsilon_{2} \sigma_{23}^{\prime} & 1 \\
\varepsilon_{1} \sigma_{14}^{\prime} & \omega_{13} & \varepsilon_{1} \sigma_{12}^{\prime} & \varepsilon_{3} \sigma_{34}^{\prime} & 1 & \varepsilon_{3} \sigma_{23}^{\prime} \\
\varepsilon_{1} \sigma_{13}^{\prime} & \varepsilon_{1} \sigma_{12}^{\prime} & \omega_{14} & 1 & \varepsilon_{4} \sigma_{34}^{\prime} & \varepsilon_{4} \sigma_{24}^{\prime} \\
\varepsilon_{2} \sigma_{24}^{\prime} & \varepsilon_{3} \sigma_{34}^{\prime} & 1 & \omega_{23} & \varepsilon_{2} \sigma_{12}^{\prime} & \varepsilon_{3} \sigma_{13}^{\prime} \\
\varepsilon_{2} \sigma_{23}^{\prime} & 1 & \varepsilon_{4} \sigma_{34}^{\prime} & \varepsilon_{2} \sigma_{12}^{\prime} & \omega_{24} & \varepsilon_{4} \sigma_{14}^{\prime} \\
1 & \varepsilon_{3} \sigma_{23}^{\prime} & \varepsilon_{4} \sigma_{24}^{\prime} & \varepsilon_{3} \sigma_{13}^{\prime} & \varepsilon_{4} \sigma_{14}^{\prime} & \omega_{34}
\end{array}\right)
$$

where

$$
\omega_{k l}:=\frac{\sigma_{i k}^{\prime} \sigma_{j l}^{\prime}+\varepsilon_{l} \sigma_{i l}^{\prime} \sigma_{j l}^{\prime} \sigma_{k l}^{\prime}+\sigma_{i l}^{\prime} \sigma_{j k}^{\prime}+\varepsilon_{k} \sigma_{i k}^{\prime} \sigma_{j k}^{\prime} \sigma_{k l}^{\prime}}{\sigma_{k l}^{2}} .
$$

Proof. We compute the respective derivatives, that constitute the entries of $\operatorname{Jac}^{\star}(T)$. Suppose that $\varepsilon_{i} \neq 0, i \in\{1,2,3,4\}$, since the cases when $\varepsilon_{j}=0$ for some $j \in\{1,2,3,4\}$ can be dealt with in an analogous manner. Then for $\{i, j, k, l\}=\{1,2,3,4\}$, one has

$$
\begin{gathered}
\frac{\partial \ell_{k l}}{\partial a_{i j}}=\frac{\partial \ell_{k l}}{\partial b_{k l}^{j}} \frac{\partial b_{k l}^{j}}{\partial a_{i j}}=-\varepsilon_{i} \frac{\mu_{k l}^{j}}{M^{j}} \cdot \varepsilon_{i} \frac{\sin a_{i j}}{M_{j k l}} \overbrace{=}^{(1)}-\frac{1}{M^{j}} \frac{\sin a_{i j}}{\sin a_{i k} \sin a_{i l}}= \\
-\frac{1}{M^{j}} \frac{\sin a_{i j}}{\sin a_{i k} \sin a_{i l}} \frac{1}{\sigma_{i j}} \frac{1}{\sigma_{k l}} \sigma_{i j} \sigma_{k l} \overbrace{=}^{(2)}-\frac{1}{M^{j}} \frac{\sin a_{i j}}{\sin a_{i k} \sin a_{i l}} \sigma_{i j} \sigma_{k l} \frac{\mu_{j k}^{i} \mu_{j l}^{i}}{M^{i}} \frac{\mu_{i k}^{l} \mu_{j k}^{l}}{M^{l}} \overbrace{=}^{(3)}
\end{gathered}
$$




$$
-\frac{M_{i j k} M_{i k l} M_{i j l} M_{j k l}}{\sqrt{(-\operatorname{det} G)^{3}}} \sigma_{i j} \sigma_{k l} \overbrace{=}^{(4)}-\sqrt{\frac{\Pi_{i=1}^{4} \varepsilon_{i} \operatorname{det} G_{i i}}{(-\operatorname{det} G)^{3}}} \sigma_{i j} \sigma_{k l}=-\eta \sigma_{i j} \sigma_{k l} .
$$

Here we used the definitions of vertex and face momenta, as well as Lemmata 1 and 4. Indeed, in (1) we have that $M_{i j k}=\mu_{k l}^{j} \sin a_{i k} \sin a_{i l}$ and in (2) we use the fact that $\sigma_{i j}=\frac{\mu_{i k}^{l} \mu_{j k}^{l}}{M^{l}}, \sigma_{k l}=\frac{\mu_{j k}^{i} \mu_{j l}^{i}}{M^{i}}$. In (3) we use

$$
\begin{aligned}
& \mu_{j k}^{i}=\frac{M_{i j k}}{\sin a_{j l} \sin a_{k l}}, \quad \mu_{i k}^{l}=\frac{M_{i k l}}{\sin a_{i j} \sin a_{j k}}, \\
& \mu_{j l}^{i}=\frac{M_{i j l}}{\sin a_{j k} \sin a_{k l}}, \quad \mu_{j k}^{l}=\frac{M_{j k l}}{\sin a_{i j} \sin a_{i k}},
\end{aligned}
$$

together with the identities of Lemma 4 . In (4) we use Lemma 3. Analogous to the above, we compute for $\{i, j, k, l\}=\{1,2,3,4\}$,

$$
\begin{gathered}
\frac{\partial \ell_{k l}}{\partial a_{i k}}=\frac{\partial \ell_{k l}}{\partial b_{j k}^{i}} \frac{\partial b_{j k}^{i}}{\partial a_{i k}}=-\varepsilon_{k} \frac{\sin a_{i k}}{M_{i j l}} \frac{\mu_{k l}^{i}}{M^{i}} \sigma_{j k}^{\prime}= \\
-\varepsilon_{k} \frac{\sqrt{\varepsilon_{j} \operatorname{det} G_{j j}}}{M^{i} \sin a_{j k} \sin a_{j l}} \frac{\sin a_{i k}}{\sqrt{\varepsilon_{k} \operatorname{det} G_{k k}}} \frac{1}{\sigma_{i k} \sigma_{k l}} \sigma_{i k} \sigma_{k l} \sigma_{j k}^{\prime}= \\
-\varepsilon_{k} \frac{\sqrt{\varepsilon_{j} \operatorname{det} G_{j j}}}{M^{i} \sin a_{j k} \sin a_{j l}} \frac{\sin a_{i k}}{\sqrt{\varepsilon_{k} \operatorname{det} G_{k k}}} \frac{\mu_{j k}^{i} \mu_{j l}^{i}}{M^{i}} \frac{\mu_{i l}^{j}}{M_{k l}^{j}} \sigma_{i k} \sigma_{k l} \sigma_{j k}^{\prime}= \\
-\varepsilon_{k} \frac{\sqrt{\varepsilon_{j} \operatorname{det} G_{j j}}}{\sqrt{\varepsilon_{k} \operatorname{det} G_{k k}}} \frac{\sqrt{\varepsilon_{l} \operatorname{det} G_{l l}} \sqrt{\varepsilon_{k} \operatorname{det} G_{k k}} \sqrt{\varepsilon_{k} \operatorname{det} G_{k k}} \sqrt{\varepsilon_{i} \operatorname{det} G_{i i}}}{\sqrt{(-\operatorname{det} G)^{3}}} \sigma_{i k} \sigma_{k l} \sigma_{j k}^{\prime}= \\
-\varepsilon_{k} \sqrt{\frac{\Pi_{i=1}^{4} \varepsilon_{i} \operatorname{det} G_{i i}}{(-\operatorname{det} G)^{3}} \sigma_{i k} \sigma_{k l} \sigma_{j k}^{\prime}=-\varepsilon_{k} \eta \sigma_{i k} \sigma_{k l} \sigma_{j k}^{\prime} .}
\end{gathered}
$$

Finally, for $\{i, j, k, l\}=\{1,2,3,4\}$, we compute the derivative

$$
\frac{\partial \ell_{k l}}{\partial a_{k l}}=\frac{\partial \ell_{k l}}{\partial b_{j k}^{i}} \frac{\partial b_{j k}^{i}}{\partial a_{k l}}+\frac{\partial \ell_{k l}}{\partial b_{j l}^{i}} \frac{\partial b_{j l}^{i}}{\partial a_{k l}} .
$$

Since the two terms of the above sum are symmetric under the permutation of $k$ and $l$, we may compute only the first one. The second one will be analogous. By Lemmata 1 and 2, we get

$$
\frac{\partial \ell_{k l}}{\partial b_{j k}^{i}} \frac{\partial b_{j k}^{i}}{\partial a_{k l}}=-\varepsilon_{l} \frac{\mu_{k l}^{i}}{M^{i}} \frac{\sin a_{i l}}{M_{i j k}} \sigma_{j l}^{\prime} \mu_{i k}^{\prime j} \overbrace{=}^{(5)}
$$




$$
\begin{gathered}
-\left(\sigma_{i k}^{\prime} \sigma_{j l}^{\prime}+\varepsilon_{l} \sigma_{i l}^{\prime} \sigma_{j l}^{\prime} \sigma_{k l}^{\prime}\right) \frac{\mu_{k l}^{i}}{M^{i}} \frac{\sin a_{i l}}{M_{i j k}} \frac{1}{\sigma_{i l} \sigma_{k l}} \overbrace{=}^{(6)} \\
-\left(\sigma_{i k}^{\prime} \sigma_{j l}^{\prime}+\varepsilon_{l} \sigma_{i l}^{\prime} \sigma_{j l}^{\prime} \sigma_{k l}^{\prime}\right) \frac{\sqrt{\varepsilon_{j} \operatorname{det} G_{j j}}}{M^{i} \sin a_{j k} \sin a_{j l}} \frac{\sin a_{i l}}{\sqrt{\varepsilon_{l} \operatorname{det} G_{l l}}} \frac{\mu_{j k}^{i} \mu_{j l}^{i}}{M^{i}} \frac{\mu_{i k}^{j}}{M_{k l}^{j}} \overbrace{=}^{(7)} \\
-\left(\sigma_{i k}^{\prime} \sigma_{j l}^{\prime}+\varepsilon_{l} \sigma_{i l}^{\prime} \sigma_{j l}^{\prime} \sigma_{k l}^{\prime}\right) \sqrt{\frac{\varepsilon_{j} \operatorname{det} G_{j j}}{(-\operatorname{det})^{3}}} \frac{\sqrt{\varepsilon_{l} \operatorname{det} G_{l l}} \sqrt{\varepsilon_{k} \operatorname{det} G_{k k}} \sqrt{\varepsilon_{l} \operatorname{det} G_{l l}} \sqrt{\varepsilon_{i} \operatorname{det} G_{i i}}}{\sqrt{\varepsilon_{l} \operatorname{det} G_{l l}}}= \\
-\left(\sigma_{i k}^{\prime} \sigma_{j l}^{\prime}+\varepsilon_{l} \sigma_{i l}^{\prime} \sigma_{j l}^{\prime} \sigma_{k l}^{\prime}\right) \sqrt{\frac{\Pi_{i=1}^{4} \varepsilon_{i} \operatorname{det} G_{i i}}{(-\operatorname{det} G)^{3}}}=-\eta\left(\sigma_{i k}^{\prime} \sigma_{j l}^{\prime}+\varepsilon_{l} \sigma_{i l}^{\prime} \sigma_{j l}^{\prime} \sigma_{k l}^{\prime}\right) .
\end{gathered}
$$

Here, in (5) we used the second Cosine Law for faces and in (6) we used the equality $M_{i k l}=\mu_{k l}^{i} \sin a_{j k} \sin a_{j l}$ together with Lemma 3. In (7) we perform a computation analogous to (3).

Thus, we obtain

$$
\begin{gathered}
\frac{\partial \ell_{k l}}{\partial a_{k l}}=\frac{\partial \ell_{k l}}{\partial b_{j k}^{i}} \frac{\partial b_{j k}^{i}}{\partial a_{k l}}+\frac{\partial \ell_{k l}}{\partial b_{j l}^{i}} \frac{\partial b_{j l}^{i}}{\partial a_{k l}}= \\
-\eta\left(\sigma_{i k}^{\prime} \sigma_{j l}^{\prime}+\varepsilon_{l} \sigma_{i l}^{\prime} \sigma_{j l}^{\prime} \sigma_{k l}^{\prime}\right)-\eta\left(\sigma_{i l}^{\prime} \sigma_{j k}^{\prime}+\varepsilon_{k} \sigma_{i k}^{\prime} \sigma_{j k}^{\prime} \sigma_{k l}^{\prime}\right)=-\eta \omega_{k l} \sigma_{k l}^{2} .
\end{gathered}
$$

The proof is completed.

\section{Dual Jacobian of a doubly truncated hyperbolic tetrahedron}

Let us consider the case when $T$ is a (mildly) doubly truncated tetrahedron depicted in Fig. 2 with dihedral angles $\theta_{i}$ and edge lengths $\ell_{i}$, $i \in\{1,2,3,4,5,6\}$. We suppose that the vertices cut off by the respective polar planes are $\mathrm{v}_{1}$ and $\mathrm{v}_{2}$.

If $T$ is mildly truncated then the formula from Theorem 6 applies. If $T$ is a prism truncated tetrahedron, as in Fig. 3, with dihedral angles $\mu, \theta_{i}$ and edge lengths $\ell, \ell_{i}, i \in\{1,2,3,5,6\}$ then its Gram matrix is given by

$$
G=\left(\begin{array}{cccc}
1 & -\cos \theta_{1} & -\cos \theta_{5} & -\cos \theta_{3} \\
-\cos \theta_{1} & 1 & -\cos \theta_{6} & -\cos \theta_{2} \\
-\cos \theta_{5} & -\cos \theta_{6} & 1 & -\cosh \ell \\
-\cos \theta_{3} & -\cos \theta_{2} & -\cosh \ell & 1
\end{array}\right)
$$

which is a slightly different notation compared to $[12,13]$.

Each link $L\left(\mathrm{v}_{k}\right), k=1,2$, is a hyperbolic quadrilateral with two right sameside angles, which can be seen as a hyperbolic triangle with a single truncated 


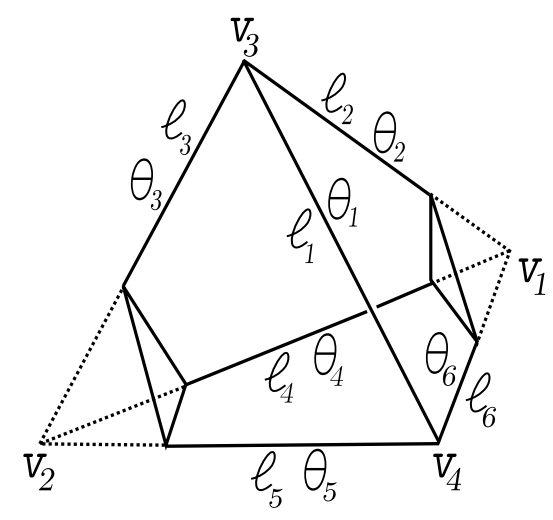

Figure 2: Doubly truncated tetrahedron (mild truncation)

vertex. Each link $L\left(\mathrm{v}_{k}\right), k=3,4$, is a spherical triangle. In the definitions of Section 2 we change each $b_{1 j}^{i}$, with $i, j \in\{2,3,4\}, i \neq j$, for $b_{1 j}^{i}+\sqrt{-1} \frac{\pi}{2}$ and each $b_{2 j}^{i}$, with $i, j \in\{1,3,4\}, i \neq j$, for $b_{2 j}^{i}+\sqrt{-1} \frac{\pi}{2}$. Thus, some of the vertex and face momenta become complex numbers. All the trigonometric rules of Section 2 still hold grace to [2, Section 4.3]. Computing the respective derivatives in a complete analogy to the proof of Theorem 6 , we obtain the following statement.

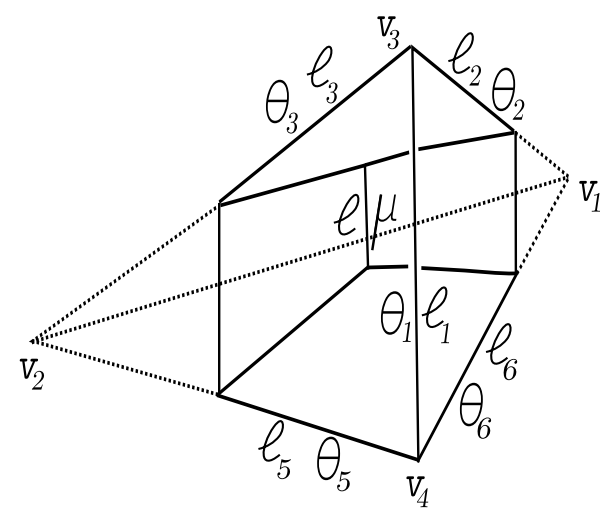

Figure 3: Doubly truncated tetrahedron (prism truncation)

Theorem 7 Let $T$ be a prism truncated tetrahedron depicted in Fig. 3. Then 
by means of the analytic continuation $a_{12}:=\sqrt{-1} \ell, \ell_{12}=\sqrt{-1} \mu$ we have

$$
\operatorname{Jac}^{\star}(T):=\frac{\partial\left(\mu, \ell_{1}, \ell_{2}, \ell_{3}, \ell_{5}, \ell_{6}\right)}{\partial\left(\ell, \theta_{1}, \theta_{2}, \theta_{3}, \theta_{5}, \theta_{6}\right)}=\frac{\partial\left(\ell_{12}, \ell_{34}, \ell_{13}, \ell_{23}, \ell_{24}, \ell_{14}\right)}{\partial\left(a_{12}, a_{34}, a_{13}, a_{23}, a_{24}, a_{14}\right)} .
$$

\section{Volume of a hyperbolic prism}

Let $\vec{\alpha}_{n}$ denote the $n$-tuple $\left(\alpha_{1}, \ldots, \alpha_{n}\right)$ with $0<\alpha_{k}<\pi, k=1, \ldots, n$. Let $\vec{\beta}_{n}$ and $\vec{\gamma}_{n}$ be analogous $n$-tuples. Let $\Pi_{n}:=\Pi_{n}\left(\vec{\alpha}_{n}, \vec{\beta}_{n}, \vec{\gamma}_{n}\right)$ be the hyperbolic $n$-sided prism depicted in Fig. 4 , with the respective dihedral angles, as shown in the picture.

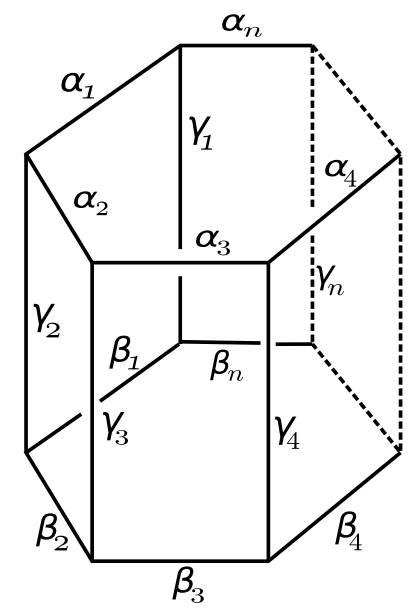

Figure 4: The prism $\Pi_{n}\left(\vec{\alpha}_{n}, \vec{\beta}_{n}, \vec{\gamma}_{n}\right)$

Let $S_{k}, k=1, \ldots, n$, be the supporting hyperplane for the $k$-th side face of the prism $\Pi_{n}$ (we start numbering the faces anti-clockwise from the side face adjacent to the angles $\alpha_{1}, \beta_{1}$ and $\gamma_{1}, \gamma_{2}$ ), and let $S_{0}$ and $S_{n+1}$ be those of the top and the bottom face, correspondingly. For each $S_{k}, k=0, \ldots, n+1$, let $S_{k}^{+}$be the respective half-space containing the unit outer normal to it. Let $S_{k}^{-}=\mathbb{H}^{3} \backslash S_{k}^{+}$. Then $\Pi_{n}=\bigcap_{i=0}^{n+1} S_{i}^{-}$.

Let $T:=T\left(\alpha, \alpha^{\prime}, \beta, \beta^{\prime}, \gamma ; \ell\right)$ be the prism truncated tetrahedron depicted in Fig. 5. Here $\alpha, \alpha^{\prime}, \beta, \beta^{\prime}$ and $\gamma$ are the respective dihedral angles, $\ell$ is the length of the respective edge. The volume $\operatorname{Vol} T$ of the tetrahedron $T$ is given by $[12 \text {, Theorem } 1]^{*}$. Let $v\left(\alpha, \alpha^{\prime}, \beta, \beta^{\prime}, \gamma ; \ell\right):=\operatorname{Vol} T\left(\alpha, \alpha^{\prime}, \beta, \beta^{\prime}, \gamma ; \ell\right)$ denote the respective volume function.

*in Section 7 we give a simplified formula for the volume of $T$. 


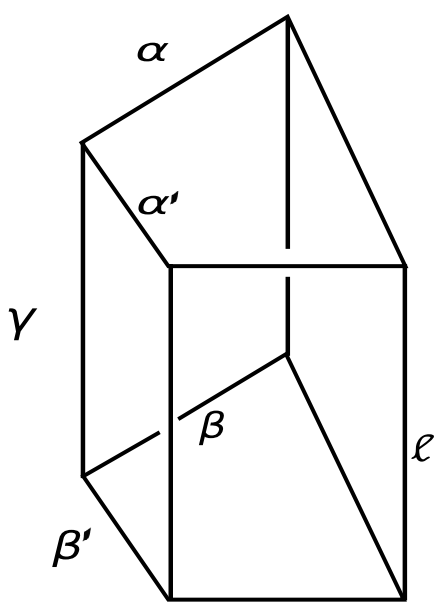

Figure 5: The prism truncated tetrahedron $T\left(\alpha, \alpha^{\prime}, \beta, \beta^{\prime}, \gamma ; \ell\right)$

Let $p_{0} p_{n+1}$ be the common perpendicular to $S_{0}$ and $S_{n+1}$. Let also define $k \oplus m:=(k+m) \bmod n$, for $k, m \in \mathbb{N}$. Then we can state the main theorem of this section.

Theorem 8 Let $\Pi_{n}=\Pi_{n}\left(\vec{\alpha}_{n}, \vec{\beta}_{n}, \vec{\gamma}_{n}\right)$ be a hyperbolic $n$-sided prism, as in Fig. 4. If $p_{0} p_{n+1} \subset \Pi_{n}$, then the volume of $\Pi_{n}$ is given by the formula

$$
\operatorname{Vol} \Pi_{n}=\sum_{k=1}^{n} v\left(\alpha_{k}, \alpha_{k \oplus 1}, \beta_{k}, \beta_{k \oplus 1}, \gamma_{k \oplus 1} ; \ell^{\star}\right),
$$

where $\ell^{\star}$ is the unique solution to the equation $\frac{\partial \Phi}{\partial \ell}(\ell)=0$, with

$$
\Phi(\ell):=\pi \ell+\sum_{k=1}^{n} v\left(\alpha_{k}, \alpha_{k \oplus 1}, \beta_{k}, \beta_{k \oplus 1}, \gamma_{k \oplus 1} ; \ell\right) .
$$

Let $P_{k}, k=1, \ldots, n$, be the plane containing $p_{0} p_{n+1}$ and orthogonal to $S_{k}$. First, we consider the case when $p_{0} p_{n+1}$ lies inside the prism $\Pi_{n}$ and the planes $P_{k}, k=1, \ldots, n$, divide the prism $\Pi_{n}$ into $n$ prism truncated tetrahedra, as shown in Fig. 6.

Then each $P_{k}$ meets the $k$-th side face of the prism $\Pi_{n}$. Thus, the planes $S_{0}, S_{k}, S_{k \oplus 1}$ and $S_{n+1}$ together with $P_{k}$ and $P_{k \oplus 1}$ become the supporting planes for the faces of a prism truncated tetrahedron, which we denote by $T_{k}$. Each $P_{k}$ is orthogonal to $S_{k}, S_{0}$ and $S_{n+1}$. The dihedral angles of 

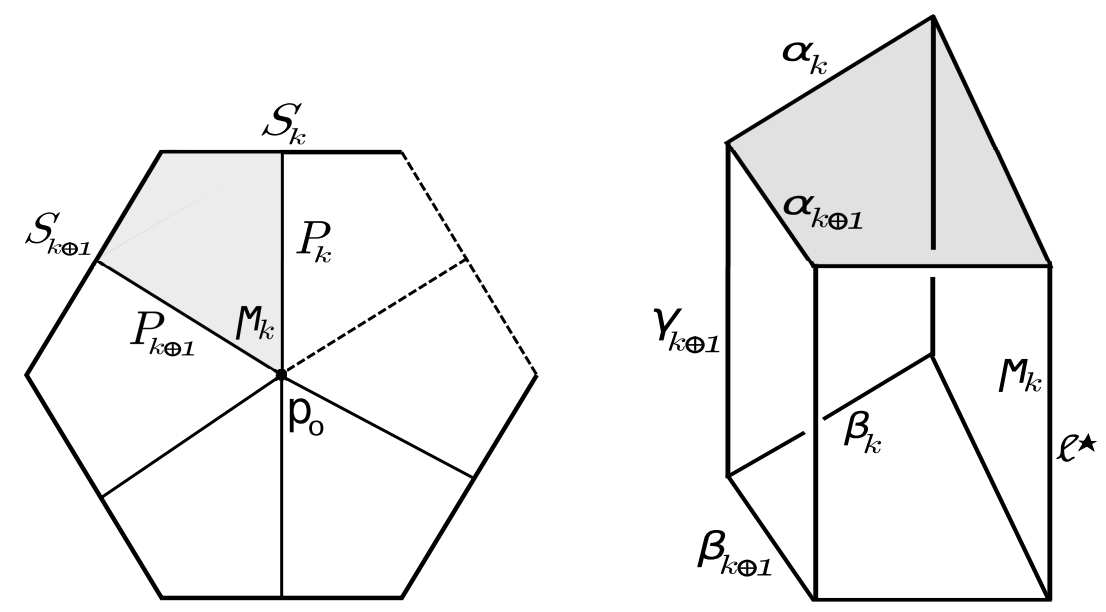

Figure 6: The decomposition of $\Pi_{n}$ (top view, on the left) and the prism truncated tetrahedron $T_{k}$ (on the right)

$T_{k}$ inherited from the prism $\Pi_{n}$ are easily identifiable. Let $\mu_{k}$ denote the dihedral angle along the edge $p_{0} p_{n+1}$ and let $\ell^{\star}$ be its length. Then we have $T_{k}=T\left(\alpha_{k}, \alpha_{k \oplus 1}, \beta_{k}, \beta_{k \oplus 1}, \gamma_{k \oplus 1} ; \ell^{\star}\right), k=1, \ldots, n$. Clearly,

$$
\operatorname{Vol} \Pi_{n}=\sum_{k=1}^{n} \operatorname{Vol} T_{k}=\sum_{k=1}^{n} v\left(\alpha_{k}, \alpha_{k \oplus 1}, \beta_{k}, \beta_{k \oplus 1}, \gamma_{k \oplus 1} ; \ell^{\star}\right) .
$$

Thus, we have to prove only the following statement.

Proposition 1 If the common perpendicular $p_{0} p_{n+1}$ is inside the prism $\Pi_{n}$ and each $P_{k}$ meets the respective side also inside $\Pi_{n}, k=1, \ldots, n$, then the equation $\frac{\partial \Phi}{\partial \ell}=0$ has a unique solution $\ell=\ell^{\star}$, the length of $p_{0} p_{n+1}$.

Proof. Let us consider the collection of prism truncated tetrahedra $T_{k}=$ $T\left(\alpha_{k}, \alpha_{k \oplus 1}, \beta_{k}, \beta_{k \oplus 1}, \gamma_{k \oplus 1} ; \ell\right), k=1, \ldots, n$. Each pair $\left\{T_{k}, T_{k \oplus 1}\right\}$ of them has an isometric face corresponding to the plane $P_{k \oplus 1}$. Indeed, each such face is completely determined by the plane angles (two right angles at the side of length $\ell$, the angles $\alpha_{k}$ and $\beta_{k}$ at the opposite side) and one side length. We obtain the prism $\Pi_{n}\left(\vec{\alpha}_{n}, \vec{\beta}_{n}, \vec{\gamma}_{n}\right)$ by glueing the tetrahedra $T_{k}$ together along the faces $P_{k}, k=1, \ldots, n$, in the respective order. Their edges of length $\ell$ match together, and one obtains a prism if the angle sum 
of the dihedral angles $\mu_{k}, k=1, \ldots, n$, along them equals $2 \pi$. We have that

$$
\frac{\partial \Phi}{\partial \ell}=\pi+\sum_{k=1}^{n} \frac{\partial v}{\partial \ell}\left(\alpha_{k}, \alpha_{k \oplus 1}, \beta_{k}, \beta_{k \oplus 1}, \gamma_{k \oplus 1} ; \ell\right) .
$$

Since $v$ is the volume function from [12, Theorem 1], then by applying the Schläfli formula [16, Equation 1] one obtains

$$
\frac{\partial \Phi}{\partial \ell}=\pi-\frac{1}{2} \sum_{k=1}^{n} \mu_{k} .
$$

Thus, whenever the tetrahedra $T_{k}$ constitute a prism, we have $\sum_{k=1}^{n} \mu_{k}=2 \pi$ or, equivalently, $\frac{\partial \Phi}{\partial \ell}=0$. The length $\ell$ in this case is exactly the length of the common perpendicular $p_{0} p_{n+1}$ to the planes $S_{0}$ and $S_{n+1}$.

The rest is to prove that $\ell=\ell^{\star}$ is a unique solution. In order to do so, we shall show that $\frac{\partial \mu_{k}}{\partial \ell}>0, k=1, \ldots, n$. By using Theorem 7 we get the following formulae for a prism truncated tetrahedron (as depicted in Fig. 3):

$$
\begin{array}{ll}
\frac{\partial \ell_{2}}{\partial \ell}=-\eta \sin \mu_{k} \sinh \ell_{6} \cosh \ell_{2}, & \frac{\partial \ell_{3}}{\partial \ell}=-\eta \sin \mu_{k} \sinh \ell_{5} \cosh \ell_{3}, \\
\frac{\partial \ell_{5}}{\partial \ell}=-\eta \sin \mu_{k} \sinh \ell_{3} \cosh \ell_{5}, & \frac{\partial \ell_{6}}{\partial \ell}=-\eta \sin \mu_{k} \sinh \ell_{2} \cosh \ell_{6} .
\end{array}
$$

Note that the above derivatives are all negative. In our present notation it means that for each prism truncated tetrahedron $T_{k}, k=$ $1, \ldots, n$, the edges of the top and bottom faces inherited from the prism $\Pi_{n}$ diminish their length if we increase solely the parameter $\ell$. Recall that $T_{k}=T\left(\alpha_{k}, \alpha_{k \oplus 1}, \beta_{k}, \beta_{k \oplus 1}, \gamma_{k \oplus 1} ; \ell\right)$, and let us denote $T_{k}^{\prime}:=$ $T\left(\alpha_{k}, \alpha_{k \oplus 1}, \beta_{k}, \beta_{k \oplus 1}, \gamma_{k \oplus 1} ; \ell^{\prime}\right)$ with $\ell^{\prime}>\ell$.

Let $A B C D$ be the top (equiv., bottom) face of $T_{k}$, as shown in Fig. 7, and $A^{\prime} B^{\prime} C^{\prime} D^{\prime}$ be the top (equiv., bottom) face of $T_{k}^{\prime}$. Since the dihedral angles accept for $\mu_{k}$ and $\mu_{k}^{\prime}$ remain the same, the plane angles of $A B C D$ at $A, B$, $C$ and those of $A^{\prime} B^{\prime} C^{\prime} D^{\prime}$ at $A^{\prime}, B^{\prime}$ and $C^{\prime}$ are respectively equal. One sees easily that we can match then $A B C D$ and $A^{\prime} B^{\prime} C^{\prime} D^{\prime}$ such that $B$ and $B^{\prime}$ coincide, the sides $A B$ and $A^{\prime} B^{\prime}, B C$ and $B^{\prime} C^{\prime}$ overlap and the point $D^{\prime}$ lies inside the quadrilateral $A B C D$. Then the area of $A^{\prime} B^{\prime} C^{\prime} D^{\prime}$ is less than that of $A B C D$. Equivalently, by the angle defect formula [1, Theorem 1.1.7], $\mu_{k}^{\prime}>\mu_{k}$. Thus, $\frac{\partial \mu_{k}}{\partial \ell}>0, k=1, \ldots, n$, and the proposition follows.

However, there is a possibility that, although the common perpendicular $p_{0} p_{n+1}$ is entirely inside the prism $\Pi_{n}$, one (or several) of the planes $P_{k}$ meets the respective $S_{k}$ partially outside of the face $S_{k}$. 


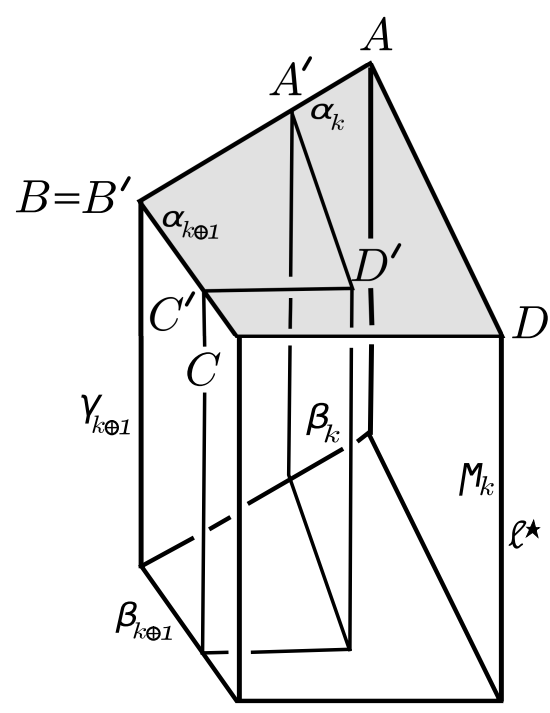

Figure 7: Prisms $T_{k}$ and $T_{k}^{\prime}$ with top faces marked

First we consider the case when a single plane $P_{k}$ meets $S_{k}$ entirely outside, as depicted in Fig. 8. Like this, we obtain the figure shaded in grey, that consists of two triangular prisms sharing an edge.

Second we consider the case when a single plane $P_{k}$ meets $S_{k}$ partially outside, as depicted in Fig. 9. Like this, we obtain a more complicated figure that consists of two tetrahedra sharing an edge (one of which has two truncated vertices).

Thus the planes $S_{0}, P_{k}, P_{k \oplus 1}, S_{k}, S_{k \oplus 1}$ and $S_{n+1}$ bound a "butterfly" prism. We put $k=1$, for clarity. In the general case, $k \geq 2$, one uses induction on the number of planes $P_{k}$ meeting $S_{k}$ outside of $\Pi_{n}$. Here, some other cases of "butterfly" prisms are possible.

Proposition 2 If the common perpendicular $p_{0} p_{n+1}$ is completely inside the prism $\Pi_{n}$, the plane $P_{1}$ meets the plane $S_{1}$ outside of $\Pi_{n}$, and all other $P_{k}$, $k=2, \ldots, n$, meet the respective side faces inside $\Pi_{n}$, then the volume of the prism equals

$$
\operatorname{Vol} \Pi_{n}=\sum_{k=1}^{n} v\left(\alpha_{k}, \alpha_{k \oplus 1}, \beta_{k}, \beta_{k \oplus 1}, \gamma_{k \oplus 1} ; \ell^{\star}\right),
$$



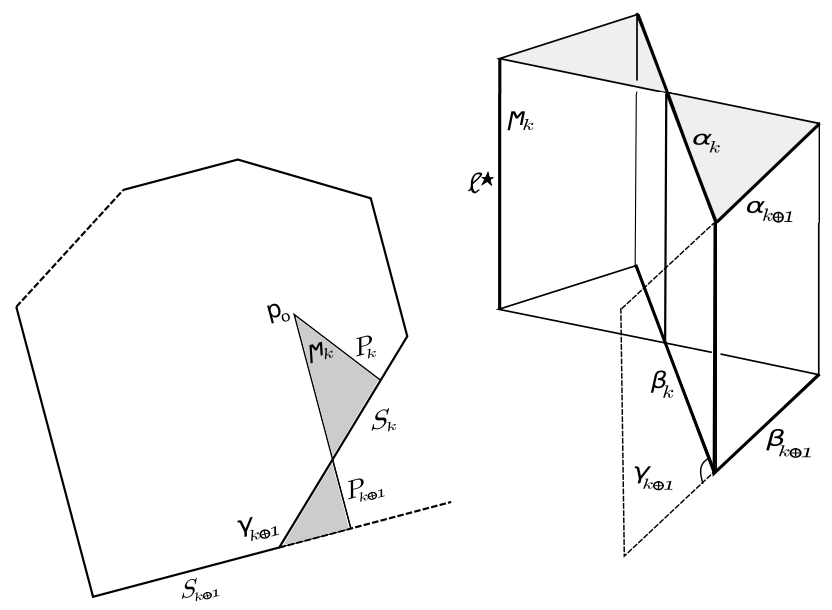

Figure 8: The decomposition of $\Pi_{n}$ (top view, on the left) and the "butterfly" prism truncated tetrahedron $T_{k}$ (on the right)

where $\ell^{\star}$ is the unique solution to the equation $\frac{\partial \Phi}{\partial \ell}(\ell)=0$, with

$$
\Phi(\ell):=\pi \ell+\sum_{k=1}^{n} v\left(\alpha_{k}, \alpha_{k \oplus 1}, \beta_{k}, \beta_{k \oplus 1}, \gamma_{k} ; \ell\right) .
$$

Proof. We start with the case of a "butterfly" prism depicted in Fig. 8. Let us observe that the "butterfly" prism $T_{1}$ overlaps with the subsequent prism truncated tetrahedron $T_{2}$ exactly on its part $T_{1}^{(o)}$ outside of $\Pi_{n}$. The part of $T_{1}$ inside $\Pi_{n}$, called $T_{1}^{(i)}$, contributes to the total volume of the prism. The volume of $T_{1}^{(o)}$ is excessive in the respective volume formula and should be subtracted. In fact, we prove that

$$
v\left(\alpha_{1}, \alpha_{2}, \beta_{1}, \beta_{2}, \gamma_{2} ; \ell^{\star}\right)=V:=\operatorname{Vol} T_{1}^{(i)}-\operatorname{Vol} T_{1}^{(o)},
$$

which implies that the excess in volume brought by $T_{2}$ is eliminated by the term “- $\operatorname{Vol} T_{1}^{(o)}$ ".

In order to do so, let us denote by $\theta$ the dihedral angle along the common edge of the triangular prisms $T_{1}^{(o)}$ and $T_{1}^{(i)}$. Let $\ell_{\theta}$ be the length of this edge. Let $\gamma:=\gamma_{2}$ and let $\ell_{\gamma}$ be the length of the vertical edge with dihedral angle $\gamma$. We know that $\frac{\partial V}{\partial \gamma}=-\frac{1}{2} \ell_{\gamma}$, by the structure of the volume formula for a prism truncated tetrahedron. Indeed, the function $V$ does not correspond to the volume of a real prism truncated tetrahedron any more, however all the 


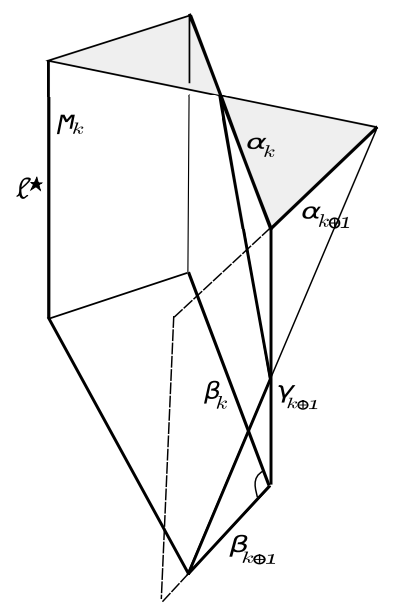

Figure 9: Another "butterfly" prism truncated tetrahedron $T_{k}$

metric relations defining the dihedral angles between the respective planes are preserved. Thus, after computing the derivative $\frac{\partial V}{\partial \ell}$ analogous to [12], we obtain the latter equality. Now we compute the respective derivatives for the parts of the "butterfly" prism $T_{1}$.

Observe that the parameter $\theta$ depends on $\gamma$, while we vary $\gamma$ and keep all other dihedral angles fixed. Let us denote $\hat{\gamma}=\pi-\gamma$ for brevity. We have that

$$
\frac{\partial \operatorname{Vol} T_{1}^{(o)}}{\partial \hat{\gamma}}=-\frac{\ell_{\gamma}}{2}-\frac{\ell_{\theta}}{2} \frac{\partial \theta}{\partial \hat{\gamma}}
$$

and

$$
\frac{\partial \operatorname{Vol} T_{1}^{(i)}}{\partial \gamma}=-\frac{\ell_{\theta}}{2} \frac{\partial \theta}{\partial \gamma},
$$

by the Schläfli formula [16, Equation 1].

The above identities together with the fact that $\frac{\partial}{\partial \hat{\gamma}}=-\frac{\partial}{\partial \gamma}$ imply that

$$
\frac{\partial}{\partial \gamma_{2}} v\left(\alpha_{1}, \alpha_{2}, \beta_{1}, \beta_{2}, \gamma_{2} ; \ell^{\star}\right)=\frac{\partial V}{\partial \gamma_{2}} .
$$

By analogy, we can prove that

$$
\frac{\partial}{\partial \xi} v\left(\alpha_{1}, \alpha_{2}, \beta_{1}, \beta_{2}, \gamma_{2} ; \ell^{\star}\right)=\frac{\partial V}{\partial \xi},
$$

for any $\xi \in\left\{\alpha_{1}, \alpha_{n}, \beta_{1}, \beta_{n}, \mu_{1}\right\}$. The volume formula for a prism truncated tetrahedron implies that by setting $\alpha_{1}=\alpha_{n}=\pi / 2$ and $\beta_{1}=\beta_{n}=\pi / 2$ we 


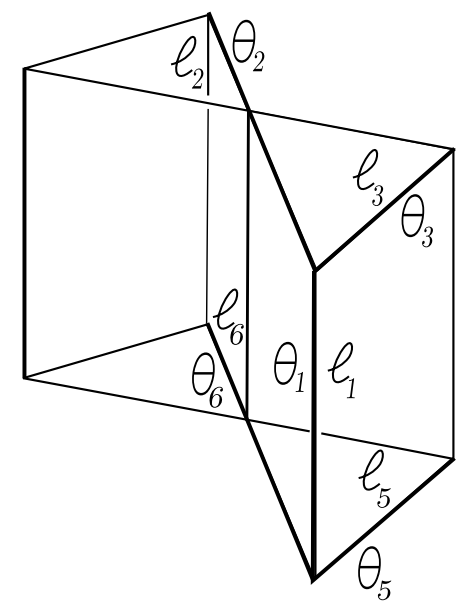

Figure 10: Parametrising the "butterfly" prism depicted in Fig. 8

get $v\left(\alpha_{1}, \alpha_{n}, \beta_{1}, \beta_{n}, \gamma_{2} ; \ell^{\star}\right)=0$. In the case of a "butterfly" prism $T_{1}$, under the same assignment of dihedral angles, we have that the bases of the two triangular prisms become orthogonal to their lateral sides. Thus $T_{1}^{(i)}$ and $T_{1}^{(o)}$ degenerate into Euclidean prisms, which means that their volumes tend to zero. Thus, we obtain the identity $v\left(\alpha_{1}, \alpha_{n}, \beta_{1}, \beta_{n}, \gamma_{2} ; \ell^{\star}\right)=V$.

The proof of the monotonicity for the function $\frac{\partial \Phi}{\partial \ell}(\ell)$ is analogous to that in Proposition 1. However, since the part $T_{1}^{(o)}$ contributes to the function $v\left(\alpha_{1}, \alpha_{n}, \beta_{1}, \beta_{n}, \gamma_{2} ; \ell\right)$ with the negative sign, we have to replace the edge lengths $\ell_{3}$ and $\ell_{5}$ with $-\ell_{3}$ and $-\ell_{5}$, respectively, as shown in Fig. 10 . Then we recompute the respective derivatives of the lengths of the horizontal edges according to Theorem 7 . We obtain that the lengths $\ell_{2}$ and $\ell_{6}$ diminish, as before, while the lengths $\ell_{3}$ and $\ell_{5}$ increase. This implies that the upper (resp., lower) triangular base of $T_{1}^{(i) \prime}$ can be placed entirely inside the upper (resp. lower) triangular base of $T_{1}^{(i)}$. By the area comparison argument, we have that $\mu_{1}^{\prime}>\mu_{1}$. The inequality $\frac{\partial \mu_{1}}{\partial \ell}>0$ follows.

All other cases of "butterfly prisms" (e.g. that in Fig. 9) can be considered by analogy.

Remark. In the general case, when the common perpendicular $p_{0} p_{n+1}$ does not lie entirely inside the prism $\Pi_{n}$, we expect that an analogue to Theorem 8 holds with an exception that the equation $\frac{\partial \Phi}{\partial \ell}(\ell)=0$ may have several solutions. However, one of these solutions is geometric and yields the volume of $\Pi_{n}$. 


\section{$7 \quad$ Modified volume formula}

We modify the volume formula for a prism truncated tetrahedron from [12], in order to reduce it to a simpler form. Indeed, the formula in [12, Theorem 1] uses analytic continuation and accounts for possible branching with respect to any variable $a_{j}=e^{\ell}$, with some $j \in\{1,2, \ldots, 6\}$, and $a_{k}=e^{i \theta_{k}}$, for any $k \in\{1,2, \ldots, 6\} \backslash\{j\}$. Usually, we put $j=4$ for simplicity. However, the formula allows for intense truncation at any edge, since it is invariant under a permutation of the variables $a_{l}, l \in\{1, \ldots, 6\}$.

In our case, given a prism $\Pi_{n}$ and its decomposition into prism truncated tetrahedra $T_{i}, i \in\{1, \ldots, n\}$, we know that only the common perpendicular $p_{0} p_{n+1}$ is produced by an intense truncation. Thus, we can always put $j=4$ and, moreover, the variable $a_{4}$ will be the only one that might cause branching.

In this case, we suggest a simplified version of the formula from [12, Theorem 1]. This formula also has less numeric discrepancies and performs faster, if used for an actual computation.

Let us put $a_{k}:=e^{i \theta_{k}}, k \in\{1,2,3,5,6\}, a_{4}:=e^{\ell}$, and let $\mathscr{U}=$ $\mathscr{U}\left(a_{1}, a_{2}, a_{3}, a_{4}, a_{5}, a_{6}, z\right)$ denote

$$
\begin{aligned}
\mathscr{U} & :=\operatorname{Li}_{2}(z)+\operatorname{Li}_{2}\left(a_{1} a_{2} a_{4} a_{5} z\right)+\operatorname{Li}_{2}\left(a_{1} a_{3} a_{4} a_{6} z\right)+\operatorname{Li}_{2}\left(a_{2} a_{3} a_{5} a_{6} z\right) \\
& -\operatorname{Li}_{2}\left(-a_{1} a_{2} a_{3} z\right)-\operatorname{Li}_{2}\left(-a_{1} a_{5} a_{6} z\right)-\operatorname{Li}_{2}\left(-a_{2} a_{4} a_{6} z\right)-\operatorname{Li}_{2}\left(-a_{3} a_{4} a_{5} z\right),
\end{aligned}
$$

where $\operatorname{Li}_{2}(\circ)$ is the dilogarithm function.

Let $z_{-}$and $z_{+}$be two solutions to the equation $e^{z \frac{\partial \mathscr{U}}{\partial z}}=1$ in the variable $z$. According to $[12,20]$, these are

$$
z_{-}:=\frac{-q_{1}-\sqrt{q_{1}^{2}-4 q_{0} q_{2}}}{2 q_{2}} \text { and } z_{+}:=\frac{-q_{1}+\sqrt{q_{1}^{2}-4 q_{0} q_{2}}}{2 q_{2}}
$$

where

$$
\begin{array}{r}
q_{0}:=1+a_{1} a_{2} a_{3}+a_{1} a_{5} a_{6}+a_{2} a_{4} a_{6}+a_{3} a_{4} a_{5}+a_{1} a_{2} a_{4} a_{5}+a_{1} a_{3} a_{4} a_{6}+a_{2} a_{3} a_{5} a_{6}, \\
q_{1}:=-a_{1} a_{2} a_{3} a_{4} a_{5} a_{6}\left(\left(a_{1}-\frac{1}{a_{1}}\right)\left(a_{4}-\frac{1}{a_{4}}\right)+\left(a_{2}-\frac{1}{a_{2}}\right)\left(a_{5}-\frac{1}{a_{5}}\right)\right. \\
\left.+\left(a_{3}-\frac{1}{a_{3}}\right)\left(a_{6}-\frac{1}{a_{6}}\right)\right) \\
q_{2}:=a_{1} a_{2} a_{3} a_{4} a_{5} a_{6}\left(a_{1} a_{4}+a_{2} a_{5}+a_{3} a_{6}+a_{1} a_{2} a_{6}+a_{1} a_{3} a_{5}+a_{2} a_{3} a_{4}+\right. \\
\left.a_{4} a_{5} a_{6}+a_{1} a_{2} a_{3} a_{4} a_{5} a_{6}\right) .
\end{array}
$$




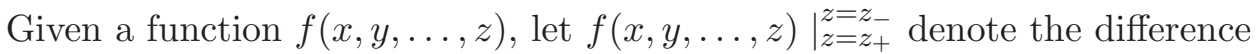
$f\left(x, y, \ldots, z_{-}\right)-f\left(x, y, \ldots, z_{+}\right)$. Now we define the following function $\mathscr{V}=$ $\mathscr{V}\left(a_{1}, a_{2}, a_{3}, a_{4}, a_{5}, a_{6}, z\right)$ by means of the equality

$$
\mathscr{V}:=\left.\frac{i}{4}\left(\mathscr{U}\left(a_{1}, a_{2}, a_{3}, a_{4}, a_{5}, a_{6}, z\right)-z \frac{\partial \mathscr{U}}{\partial z} \log z\right)\right|_{z=z_{+}} ^{z=z_{-}} .
$$

Proposition 3 The volume of a prism truncated tetrahedron $T$ is given by

$$
\operatorname{Vol} T=\Re\left(-\mathscr{V}+a_{4} \frac{\partial \mathscr{V}}{\partial a_{4}} \log a_{4}\right) .
$$

Proof. Let us denote

$$
f(T)=\Re\left(-\mathscr{V}+a_{4} \frac{\partial \mathscr{V}}{\partial a_{4}} \log a_{4}\right),
$$

and compute the derivative

$$
\begin{aligned}
& \frac{\partial}{\partial \ell}\left(f(T)+\frac{\mu \ell}{2}\right)=a_{4} \frac{\partial}{\partial a_{4}}\left(f(T)+\frac{\mu \log a_{4}}{2}\right)= \\
& =a_{4} \frac{\partial}{\partial a_{4}}\left(\Re\left(-\mathscr{V}+\left(a_{4} \frac{\partial \mathscr{V}}{\partial a_{4}}+\frac{\mu}{2}\right) \log a_{4}\right)\right) .
\end{aligned}
$$

The function $\Re\left(a_{4} \frac{\partial \mathscr{V}}{\partial a_{4}}+\frac{\mu}{2}\right)$ has an a.e. vanishing derivative, c.f. the note in [12] after Theorem 1 saying that $\mu \equiv-2 \Re\left(a_{4} \frac{\partial \mathscr{V}}{\partial a_{4}}\right) \bmod \pi$. Hence,

$$
\begin{gathered}
\frac{\partial}{\partial \ell}\left(f(T)+\frac{\mu \ell}{2}\right)=a_{4} \frac{\partial}{\partial a_{4}}\left(\Re\left(-\mathscr{V}+\left(a_{4} \frac{\partial \mathscr{V}}{\partial a_{4}}+\frac{\mu}{2}\right) \log a_{4}\right)\right) \overbrace{=}^{(1)} \\
\overbrace{=}^{(1)} \Re\left(-a_{4} \frac{\partial \mathscr{V}}{\partial a_{4}}+a_{4} \frac{\partial \mathscr{V}}{\partial a_{4}}+\frac{\mu}{2}\right)=\frac{\mu}{2} .
\end{gathered}
$$

The equality (1) holds because of the commutativity of the operations $\Re$ and $\frac{\partial}{\partial a_{4}}$ for the function $-\mathscr{V}+\left(a_{4} \frac{\partial \mathscr{V}}{\partial a_{4}}+\frac{\mu}{2}\right) \log a_{4}$. The latter holds since $a_{4}=e^{\ell}$ is a real parameter.

This implies that $\frac{\partial f(T)}{\partial \mu}=-\frac{\ell}{2}$. By analogy to the proof of [12, Theorem 1], we can show that $\frac{\partial f(T)}{\partial \theta_{k}}=-\frac{\ell_{k}}{2}$, and that if $T$ degenerates into a right Euclidean prism, then $f(T) \rightarrow 0$. Thus, $\operatorname{Vol} T=f(T)$ and the proposition follows.

Also, we have the following way to determine the dihedral angle $\mu$ along the length $\ell$ edge coming from the intense truncation. 
Proposition 4 The angle $\mu$ is given by

$$
\mu \equiv-\Re\left(\left.\frac{i a_{4}}{2} \frac{\partial \mathscr{U}\left(a_{1}, \ldots, a_{6}, z\right)}{\partial a_{4}}\right|_{z=z_{+}} ^{z=z_{-}}\right) \bmod \pi .
$$

Proof. We have $\mu \equiv-2 \Re\left(a_{4} \frac{\partial \mathscr{V}}{\partial a_{4}}\right) \bmod \pi$, where $0<\mu<\pi$ and has an a.e. vanishing derivative.

Then we compute

$$
\begin{gathered}
\frac{\partial \mathscr{U}\left(a_{1}, \ldots, a_{6}, z_{ \pm}\left(a_{1}, \ldots, a_{6}\right)\right)}{\partial a_{4}}-\frac{\partial}{\partial a_{4}}\left(z_{ \pm} \frac{\partial \mathscr{U}\left(a_{1}, \ldots, a_{6}, z_{ \pm}\right)}{\partial z} \log z_{ \pm}\right)= \\
\frac{\partial \mathscr{U}\left(a_{1}, \ldots, a_{6}, z_{ \pm}\right)}{\partial a_{4}}+\frac{\partial z_{ \pm}}{\partial a_{4}} \frac{\partial \mathscr{U}\left(a_{1}, \ldots, a_{6}, z_{ \pm}\right)}{\partial z} \\
-\frac{\partial z_{ \pm}}{\partial a_{4}} \frac{\partial \mathscr{U}\left(a_{1}, \ldots, a_{6}, z_{ \pm}\right)}{\partial z}= \\
\frac{\partial \mathscr{U}\left(a_{1}, \ldots, a_{6}, z_{ \pm}\right)}{\partial a_{4}},
\end{gathered}
$$

since, for some $m \in \mathbb{Z}$,

$$
z_{ \pm} \frac{\partial \mathscr{U}\left(a_{1}, \ldots, a_{6}, z_{ \pm}\right)}{\partial z}=2 \pi i m
$$

by the definition of $z_{-}$and $z_{+}$.

Therefore, we obtain

$$
\mu \equiv-2 \Re\left(a_{4} \frac{\partial \mathscr{V}}{\partial a_{4}}\right) \bmod \pi \equiv-\Re\left(\left.\frac{i a_{4}}{2} \frac{\partial \mathscr{U}\left(a_{1}, \ldots, a_{6}, z\right)}{\partial a_{4}}\right|_{z=z_{+}} ^{z=z_{-}}\right) \bmod \pi,
$$

where $0<\mu<\pi$.

\section{Numerical examples}

Finally, we produce some numerical examples concerning an $n$-gonal ( $n \geq 5$ ) prism $\Pi_{n}$ with the following distribution of dihedral angles: the angles along the vertical edges are $\frac{2 \pi}{5}$, the angles adjacent to the bottom face are $\frac{\pi}{3}$, and those adjacent to the top face are $\frac{\pi}{2}$. Indeed, such a prism $\Pi_{n}$ exists due to [8, Theorem 1.1]. Then we apply Theorem 8 for the cases $n=5,6,7$, and perform all necessary numeric computations with Wolfram Mathematica ${ }^{\circledR}$. 
In order to avoid excessive branching in numerical computations, we use the modified parameters

$$
q_{i}^{\prime}:=\frac{q_{i}}{\Pi_{k=1}^{6} a_{k}} \text { and } z_{ \pm}:=\frac{-q_{1}^{\prime}-\sqrt{q_{1}^{\prime 2} \pm 4 q_{0}^{\prime} q_{2}^{\prime}}}{2 q_{2}^{\prime}} .
$$

in the formulae for $\mathscr{U}$ and $\mathscr{V}$ from Section 7.

It follows from the definition of $q_{i}^{\prime}, i=1,2,3$, above that the quantity $q_{1}^{\prime 2}-4 q_{0}^{\prime} q_{2}^{\prime}$ is a real number, c.f. [20, Section 1.1 , Lemma]. This fact prevents computational discrepancies and simplifies any further numerical analysis of the volume formula.

\begin{tabular}{|c|c|c|}
\hline$n$ & $\left(\ell^{\star}, \mu\right)$ & $\operatorname{Vol}_{n}$ \\
\hline 5 & $(0.50672,2 \pi / 5)$ & 2.63200 \\
6 & $(0.38360, \pi / 3)$ & 3.43626 \\
7 & $(0.312595,2 \pi / 7)$ & 4.19077 \\
\hline
\end{tabular}

Table 1: Left: parameters $\left(\ell^{\star}, \mu\right)$ of $T_{n}$, right: volume of $\Pi_{n}$

Each of the above prisms $\Pi_{n}$ can be subdivided into $n$ isometric copies of a prism truncated tetrahedron $T_{n}$. Indeed, $T_{n}$ is a prism truncated tetrahedron with angles $\theta_{1}=\frac{2 \pi}{5}, \theta_{2}=\theta_{3}=\frac{\pi}{2}, \theta_{5}=\theta_{6}=\frac{\pi}{3}$, and $\mu=\frac{2 \pi}{n}$. By rotating it along the edge with dihedral angle $\mu$, we compose the desired prism $\Pi_{n}$.

The graph of $\operatorname{Vol} T_{n}$, with $n=5$, as a function of $\ell$, is shown in Fig. 11 on the left. The graph of $\frac{\partial \Phi}{\partial \ell}(\ell)$ for the same prism truncated tetrahedron $T_{n}$ is depicted in Fig. 11 on the right. We observe that the function $\frac{\partial \Phi}{\partial \ell}(\ell)$ is indeed monotone and has a single zero $\ell^{\star} \approx 0.50672 \ldots$.
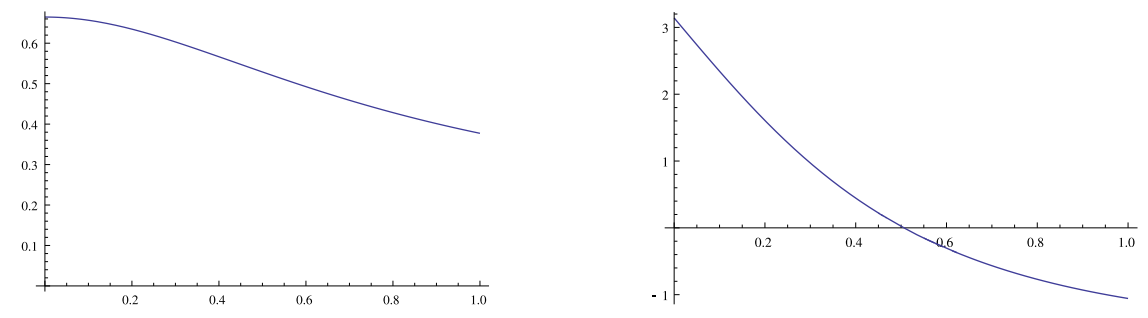

Figure 11: Left: $\operatorname{Vol} T_{5}$, right: $\frac{\partial \Phi}{\partial \ell}$, both as functions of $\ell$

The volume of $T_{5}$ with $\theta_{1}=\frac{2 \pi}{5}, \theta_{2}=\theta_{3}=\frac{\pi}{2}, \theta_{5}=\theta_{6}=\frac{\pi}{3}$ and $\ell^{\star} \approx 0.50672 \ldots$ equals $\sim 0.52639 \ldots$ by Proposition 3 . Thus, we can see that $\operatorname{Vol} \Pi_{5}=5 \cdot \operatorname{Vol} T_{5}$ in accordance with Theorem 8 , and from Proposition 4 $\mu=1.25664 \ldots \approx \frac{2 \pi}{5}$. 


\section{References}

[1] P. Buser "Geometry and spectra of compact Riemann surfaces", NewYork, Heidelberg, London: Springer, 2010.

[2] Y. Cho "Trigonometry in extended hyperbolic space and extended de Sitter space", Bull. Korean Math. Soc. 46 (6) 1099-1133 (2009); arXiv:0712.1877.

[3] Y. Chо, H. Kim "On the volume formula for hyperbolic tetrahedra", Discrete Comput. Geom. 22 (3), 347-366 (1999).

[4] D.A. Derevnin, A.C. Kim "The Coxeter prisms in $\mathbb{H}^{3}$ " in Recent advances in group theory and low-dimensional topology (Heldermann, Lemgo, 2003), pp. 35-49.

[5] D.A. Derevnin, A.D. Mednykh "A formula for the volume of a hyperbolic tetrahedron", Russ. Math. Surv., 60 (2), 346-348 (2005).

[6] W. Fenchel "Elementary geometry in hyperbolic space", Berlin, NewYork: Walter de Gruyter, 1989.

[7] R. Guo "Calculus of generalized hyperbolic tetrahedra", Geometriae Dedicata, 153 (1), 139-149 (2011); arXiv:1007.0453.

[8] C.D. Hodgson, I. Rivin "A characterization of compact convex polyhedra in hyperbolic 3-space", Invent. Math. 111, 77-111 (1993).

[9] R. Guo, F. Luo "Rigidity of polyhedral surfaces - II", Geom. Topol. 13, 1265-1312 (2009); arXiv:0711.0766.

[10] R.M. Kashaev "The hyperbolic volume of knots from the quantum dilogarithm", Lett. Math. Phys. 39 (3), 269-275 (1997); arXiv:q$\operatorname{alg} / 9601025$.

[11] R. Kellerhals "On the volume of hyperbolic polyhedra", Math. Ann., 285 (4), 541-569 (1989).

[12] A. Kolpakov, J. Murakami "Volume of a doubly truncated hyperbolic tetrahedron", Aequationes Math., 85 (3), 449-463 (2013); arXiv:1203.1061.

[13] A. Kolpakov, J. Murakami "Erratum to: Volume of a doubly truncated hyperbolic tetrahedron", Aequationes Math., 88 (1-2), 199-200 (2014). 
[14] A. Kolpakov, A. Mednykh, M. Pashkevich "Volume formula for a $\mathbb{Z}_{2}$-symmetric spherical tetrahedron through its edge lengths", Arkiv för Matematik, 51 (1), 99-123 (2013); arXiv:1007.3948.

[15] F. Luo "3-dimensional Schläfli formula and its generalization", Commun. Contemp. Math., 10, suppl. 1, 835-842 (2008); arXiv:0802.2580.

[16] J. Milnor "The Schläfli differential equality" in Collected Papers. I. Geometry (Publish or Perish, Houston, TX, 1994), pp. 281-295.

[17] J. Murakami "The volume formulas for a spherical tetrahedron", Proc. Amer. Math. Soc. 140 9, 3289-3295 (2012); arXiv:1011.2584.

[18] H. Murakami, J. Murakami "The colored Jones polynomials and the simplicial volume of a knot", Acta Math. 186 (1), 85-104 (2001); arXiv:math/9905075.

[19] J. Murakami, A. Ushijima "A volume formula for hyperbolic tetrahedra in terms of edge lengths", J. Geom. 83 (1-2), 153-163 (2005); arXiv:math/0402087.

[20] J. Murakami, M. Yano "On the volume of hyperbolic and spherical tetrahedron", Comm. Anal. Geom. 13 (2), 379-400 (2005).

[21] J.G. Ratcliffe, "Foundations of hyperbolic manifolds", New York: Springer-Verlag, 1994. (Graduate Texts in Math.; 149).

[22] A. Ushijima "A volume formula for generalised hyperbolic tetrahedra" in Mathematics and Its Applications 581 (Springer, Berlin, 2006), pp. 249-265. 


\author{
Alexander Kolpakov \\ Department of Mathematics \\ University of Toronto \\ 40 St. George Street \\ Toronto $O N$ \\ M5S $2 E_{4}$ Canada \\ kolpakov.alexander(at)gmail.com
}

Jun Murakami

Department of Mathematics

Faculty of Science and Engineering

Waseda University

3-4-1 Okubo Shinjuku-ku

169-8555 Tokyo, Japan

murakami(at)waseda.jp 